Itinéraires Itinéraires

Littérature, textes, cultures

\title{
Le découpage chapitral dans House of Cards : une stratégie narrative liée au modèle de diffusion de Netflix
}

Segmentation into Chapters in House of Cards: A Narrative Strategy Linked to the Netflix Broadcast Model

\section{Anaïs Goudmand}

\section{OpenEdition}

Journals

Édition électronique

URL : http://journals.openedition.org/itineraires/7673

DOI : $10.4000 /$ itineraires.7673

ISSN : 2427-920X

Éditeur

Pléiade

\section{Référence électronique}

Anaïs Goudmand, «Le découpage chapitral dans House of Cards : une stratégie narrative liée au modèle de diffusion de Netflix », Itinéraires [En ligne], 2020-1 | 2020, mis en ligne le 29 septembre 2020, consulté le 14 novembre 2020. URL : http://journals.openedition.org/itineraires/7673 ; DOI https://doi.org/10.4000/itineraires.7673

Ce document a été généré automatiquement le 14 novembre 2020.

\section{(c) (†) $\odot$}

Itinéraires est mis à disposition selon les termes de la licence Creative Commons Attribution - Pas d'Utilisation Commerciale - Pas de Modification 4.0 International. 


\title{
Le découpage chapitral dans House of Cards : une stratégie narrative liée au modèle de diffusion de Netflix
}

\author{
Segmentation into Chapters in House of Cards: A Narrative Strategy Linked to \\ the Netflix Broadcast Model
}

\author{
Anaïs Goudmand
}

1 Netflix est une entreprise américaine créée en 1997 qui propose du contenu audiovisuel en streaming sur Internet et qui comptait plus de 150 millions d'abonnés en 2019. Jusqu'en 2011, elle se contentait de racheter les droits des programmes diffusés. House of Cards (2013-2018), dont elle est l'unique diffuseur, est la première série originale qu'elle propose. Elle raconte les manœuvres politiques de Frank et Claire Underwood (Kevin Spacey, Robin Wright) pour accéder à la présidence des États-Unis, et s'inscrit dans une politiquce de rupture avec le mode de diffusion hertzien des séries télévisées au sein de laquelle le recours au chapitrage tient une place de choix : les épisodes d'une saison étant livrés en une seule fois, le même jour, ils apparaissent sous la forme de « chapitres » segmentant une portion de l'œuvre saisie d'emblée dans sa complétude.

2 Le choix du chapitrage des épisodes n'est cependant pas un phénomène entièrement nouveau ni limité aux séries Netflix, on le retrouve ponctuellement dans le cas de séries diffusées suivant le rythme hebdomadaire généralement adopté par les chaînes hertziennes. Contrairement à l'épisode, découpage conventionnel des séries télévisées, le chapitre est un intitulé marginal qui n'a pas d'usage stable. Il semble que la segmentation en chapitres, beaucoup plus fluctuante et beaucoup moins institutionnalisée à la télévision qu'en littérature, se répartit grosso modo entre deux fonctions, parfois complémentaires: soit elle relève d'une référence au modèle romanesque, soit elle permet d'établir des seuils narratifs supplémentaires entre les balises canoniques que sont l'épisode et la saison.

3 Un exemple de la première fonction est la série Jane The Virgin, diffusée depuis 2014 sur CW. Ici, le chapitrage renvoie au projet de l'héroïne de devenir écrivaine de romans à 
l'eau de rose et à son goût pour les telenovelas. Cette mention explicite du "chapitre " apparaît à l'image au bout de quelques minutes, dans un intertitre qui reprend une typographie de machine à écrire, et les épisodes s'achèvent sur une mention toute feuilletonesque: "to be continued». Les intertitres ont donc une valeur ironique et métanarrative, la série étant conçue comme une parodie de telenovela. On peut également citer les exemples de séries plus anciennes telles que The Survivors (ABC, 1969), The Gangster Chronicles (NBC, 1981), Murder One (ABC, 1995-1997) ou Boston Legal (FOX, 2000-2004), ou des récentes Legion (FX, 2017-), The Exorcist (Fox, 2016-), et Riverdale (The, CW 2017-), dont les chapitres sont l'équivalent des épisodes, puisqu'ils sont livrés de façon hebdomadaire et se conforment à des durées bien établies (45 minutes ou 60 minutes).

4 En ce qui concerne le second usage, le chapitre est parfois utilisé comme seuil inférieur à l'épisode dans le DVD, suivant un procédé identique à celui que l'on trouve dans les DVD de films, comme dans les menus de X-Files (Fox, 1993-2009) ou de Black Books (Channel 4, 2002-2004), où le chapitrage fait également écho au sujet de la série, qui se déroule dans une librairie. Il s'agit alors d'un découpage qui n'existe que dans le menu du DVD, aucune rupture n'apparaissant lorsque l'on regarde l'épisode en continu ${ }^{1}$. La terminologie s'avère ici volatile et beaucoup moins systématique que dans les DVD de films. De nombreux DVD de séries se passent de ce découpage interne ou proposent un lexique alternatif en utilisant le mot "scènes " (Doctor Who' ${ }^{2}$, Game of Thrones ${ }^{3}$, Breaking Bad $^{4}$, Buffy contre les vampires ${ }^{5}$.

5 Greek (ABC, 2007-2011) donne l'exemple d'un autre type de balisage chapitral : chaque saison est divisée en deux chapitres comprenant dix épisodes pour le chapitre 1, et douze épisodes pour le chapitre 2. Dans ce cas, alors que l'on considère souvent que le chapitre se distingue de l'épisode par l'absence de séparation temporelle, on assiste à une logique inverse : les épisodes sont diffusés de façon hebdomadaire, mais avec une pause beaucoup plus longue entre les chapitres. Par exemple, l'épisode 10 de la saison 1, dernier épisode du chapitre 1, est diffusé le 10 septembre 2007 et l'épisode 11, premier épisode du chapitre 2, le 24 mars 2008. Le chapitrage signale donc l'hiatus de plusieurs mois au milieu de la saison, ce qui se traduit d'ailleurs dans la gestion de l'économie narrative, la fin de l'épisode 10 constituant un seuil fort. L'hiatus intersaisonnier est une pratique très courante dans les saisons à vingt-deux ou vingtquatre épisodes, mais la matérialisation de ce seuil est généralement inexistante dans les DVD. Dans le cas de Greek, cette numérotation continue donc de faire sens une fois les épisodes rassemblés, par conséquent, elle est préservée dans le DVD (un DVD par chapitre).

6 Au sein de ces usages différenciés du chapitrage, Netflix, avec House of Cards, se distingue par la volonté d'imposer une nouvelle norme: le recours à la notion de chapitre s'inscrit dans le cadre d'un mode de diffusion spécifique (qui abolit l'intervalle hebdomadaire entre les épisodes) et de ses conséquences sur les pratiques de réception. Le public est alors libre de choisir son rythme d'actualisation, comme dans le cas d'un roman chapitré. J'étudierai la manière dont le chapitre travaille l'épisode en diluant l'effet de seuil dans House of Cards, dans le contexte de la politique de diffusion mise en œuvre par Netflix. 


\section{Une prise en compte des usages spectatoriaux}

7 Ainsi que le constate Henry Jenkins ([2006] 2013: 157), la multiplication des formes de consommation des séries, notamment avec l'apparition du magnétoscope, du téléchargement numérique, du visionnage sur iPod et du DVD, a conduit à une hybridation des modes de consommation et à une transformation de la production des contenus. La « forme de diffusion paternaliste ${ }^{6}$ " (Marshall 2009: 44, ma traduction) qui caractérisait le régime télévisuel est complétée de nos jours par le développement de la vidéo à la demande (VàD), qui offre la possibilité de maîtriser le temps d'actualisation, et donc, par exemple, d'attendre que l'intégralité des épisodes d'une saison soit disponible avant de commencer à la regarder, afin d'éviter la frustration de l'intermittence hebdomadaire. Ce type de diffusion constitue une réponse des chaînes de production à des usages illégaux (piratage, streaming) qui témoignent d'une volonté des récepteurs de gérer le rythme de visionnage. Combes évoque ainsi une " consommation délinéarisée » fondée sur un « "ramassage" de l'horizon temporel de la série» (2015: \$20), qui «se caractérise par une reprise en main par l'amateur du rythme et des conditions de sa pratique » $(2015:$ : 3). Le terme métaphorique «bingewatching ", issu du vocabulaire de l'addiction ${ }^{7}$, est passé dans le vocabulaire courant pour désigner une consommation "sur-ramassée » des épisodes, qui suppose en creux une perte de contrôle du spectateur sur son rythme de visionnage.

8 Le «modèle Netflix » correspond au franchissement d'un pas supplémentaire dans la prise en compte de ces usages par les producteurs et les diffuseurs. La chaîne a en effet instauré une rationalisation de la création des séries à partir de l'analyse de données, en utilisant les «Big data » qu'elle recueille auprès de ses usagers. Diverses données fines de consommation sont extraites (les fiches consultées par chaque spectateur, les programmes regardés, en intégralité ou non, le rythme d'actualisation, les pauses, les retours en arrière, etc.) à partir desquelles l'application d'algorithmes permet d'établir un système de prédiction des comportements et une anticipation de la demande. House of Cards, diffusée sur Netflix depuis 2013, est la première série dont la création est immédiatement liée à l'analyse de données. On peut distinguer plusieurs étapes dans sa production. D'abord, les droits de la série initiale de la BBC sont rachetés par un studio indépendant, Media Rights Capital. David Fincher, réalisateur reconnu, signale son intérêt pour le projet, qu'il souhaite produire avec Eric Roth. Puis Beau Willimon est recruté comme scénariste. L'agent de Kevin Spacey approche l'équipe début 2011, au moment où Willimon achève le script du pilote. Le projet est ensuite présenté à divers acheteurs potentiels: HBO, Showtime, AMC. Plusieurs chaînes sont intéressées, mais c'est Netflix qui obtient les droits de la série. Elle commande immédiatement deux saisons de treize épisodes, quand les autres chaînes ne s'engageaient que sur le pilote, et laisse la possibilité aux créateurs d'adapter la durée de l'épisode au contenu :

L'équipe de House of Cards, plutôt que de produire quelques épisodes, d'attendre qu'ils soient diffusés, puis de s'inquiéter d'une annulation, a bénéficié d'un engagement inédit de la part de Netflix. Vingt-six épisodes d'avances.

En effet, les analyses de données ont permis d'observer l'intérêt du public pour les films de Fincher et pour les films avec Spacey, et d'anticiper l'immense succès de la série, comme le souligne le directeur des programmes (Chief Content Officer) de Netflix, Ted Sarandos:

Netflix, qui possède 27 millions d'abonnés dans le pays et 33 millions dans le monde, a fait les calculs. Elle savait déjà qu'une bonne partie des spectateurs avait 
regardé du début à la fin les films de M. Fincher, le réalisateur de The Social Network. Et les films avec M. Spacey avaient également bien marché, de même que la version britannique de House of Cards. À partir de ces cercles d'intérêt, Netflix a été en mesure d'établir l'intersection d'un diagramme de Venn qui suggérait que l'achat de la série serait un très bon pari pour un programme original ${ }^{9}$.

Netflix table alors sur un principe de diffusion inédit. Plutôt que de livrer les épisodes suivant un rythme hebdomadaire, qui pousse les spectateurs qui souhaitent regarder l'ensemble de la saison à leur gré à attendre la fin de la diffusion, au risque de se faire "spoiler ", elle décide de livrer l'intégralité des épisodes le même jour. Dans une enquête publiée en 2015 sur son site presse, Netflix affirme ainsi que les données permettent d'établir à quel moment les spectateurs se font « harponner » par une série, ce qui signifie, selon leur définition de travail, de déterminer l'épisode à partir duquel $70 \%$ des spectateurs regardent la saison en intégralité ${ }^{10}$. Les résultats montrent qu'il ne s'agit jamais du premier épisode, mais d'un épisode ultérieur (le troisième dans le cas de House of Cards). Netflix y voit une validation de la stratégie de diffusion dans la mesure où le pilote n'est pas suffisant pour fidéliser le public.

\section{Un « film de 13 heures » : I'« hyper-feuilletonisation » signe-t-elle la fin du feuilleton ? Effets de lissage des seuils épisodiques}

En supprimant l'hiatus temporel qui sépare les épisodes, Netflix rapproche le découpage des séries du chapitrage littéraire. Le chapitre télévisuel serait donc l'épisode affranchi du rythme de diffusion hebdomadaire, qui résulterait d'une normalisation de la pratique du «binge watching». Si, pour reprendre la terminologie d'Ugo Dionne (2008), on conçoit le DVD ou les plateformes de VàD qui réunissent l'intégralité des épisodes d'une série comme le «dispositif» succédant au "paradispositif» que constituait la diffusion télévisuelle, on peut considérer que Netflix se défait de l'étape du paradispositif pour proposer d'emblée un mode de diffusion qui correspond à la fixation définitive de l'ensemble. Ainsi les épisodes d'une saison de House of Cards sont-ils d'emblée conçus comme un tout cohérent et continu, ce qui peut s'expliquer par les conditions de production particulièrement favorables dont ont bénéficié les créateurs. La première saison a souvent été désignée, par Willimon ou par Spacey par exemple, comme un "film de 13 heures " plutôt que comme une série télévisée au sens traditionnel du terme :

Le fait de savoir que nous avions 26 heures de garanties nous a permis un storytelling beaucoup plus sophistiqué et stratifié [...]. Vous n'avez pas besoin de vous vendre et de vous faire auditionner semaine après semaine. Vous n'avez même pas besoin d'y penser comme de la télévision. [...] Pour nous, c'était un film de 13 heures ${ }^{11}$.

Est-ce que regarder treize heures d'un seul ensemble cinématographique est réellement différent d'un film? Est-ce qu'on définit un film comme quelque chose qui dure deux heures ou moins ${ }^{12}$ ?

Cette évolution ne peut se concevoir que dans le contexte de ce que Vince Gilligan, créateur de Breaking Bad, désignait comme une «hyper-feuilletonnisation » autorisée par le développement des nouveaux supports :

Quand j'ai commencé sur des séries comme X-Files, la sagesse populaire disait qu'il fallait éviter la feuilletonnisation. [...] La vidéo à la demande permet une forme de récit hyper-feuilletonnant et laisse aux gens la liberté d'accéder au contenu quand ils le souhaitent ${ }^{13}$. 
Dans une représentation désormais très répandue, l'«âge d'or » des séries télévisées serait lié au retour des formes feuilletonesques, centrées sur une mise en intrigue continue, par contraste avec le modèle épisodique ${ }^{14}$. Elles sont perçues comme plus ambitieuses, plus complexes et plus exigeantes que les séries « sérielles». En outre, elles possèdent l'avantage de la longueur, dont ne disposent pas les films. C'est ici qu'intervient la référence au modèle littéraire : les séries "hyper-feuilletonnantes » offrent la possibilité quasi inédite de créer des récits audiovisuels d'ampleur romanesque. Willimon rapproche ainsi l'ambition de ce type de séries de celle d'un roman de Tolstoï :

Le récit hyper-feuilletonnant - qu'on voit depuis $\mathrm{Oz}$ - se rapproche beaucoup plus d'un roman que de quoi que ce soit d'autre. Il peut avoir la taille et l'envergure d' Anna Karénine : des centaines de personnages qui se faufilent dans l'histoire, tandis que la focalisation change selon les chapitres. Cela permet de creuser les personnages d'une manière qui était inenvisageable en 90 ou 120 minutes - que ce soit au théâtre ou à la télévision ${ }^{15}$.

Nous avons toujours conçu cela comme une sorte de film de treize heures, bien avant Netflix. Nous ne voulions pas créer une "série télévisée ", nous voulions créer une histoire monumentale de dimension épique. Malgré une organisation et une structure en épisodes ou en chapitres proches du modèle télévisuel, cela n'était pas l'approche stylistique que nous avons adoptée pendant l'écriture et le tournage ${ }^{16}$.

On peut rappeler ici l'origine littéraire de la série, inspirée de la série anglaise éponyme (1990), elle-même adaptée de la trilogie romanesque de Michael Dobbs, qui se déroule au Royaume-Uni à la fin du mandat de Margaret Thatcher ${ }^{17}$. Ce recours à l'héritage romanesque peut être interprété comme une stratégie de légitimation, mais pas uniquement: du point de vue de la réception, l'analogie se justifie également par la proximité temporelle entre l'immersion romanesque et l'immersion «hyperfeuilletonnante ", qui dépendent toutes deux de l'approfondissement des personnages et du monde narratif dans la longue durée. Le choix de l'intitulé «chapitre » pour les épisodes de House of Cards est donc loin d'être arbitraire et induit une série de conséquences poétiques.

10 Dès lors que la rupture temporelle entre les épisodes disparait, les problèmes de mémorisation se posent avec moins d'acuité, ce qui permet aux créateurs de débarrasser le récit des dispositifs de rappel et de teasing, qu'ils soient intra- ou extradiégétiques. Ted Sarandas souligne ainsi le bénéfice poétique qui peut en être tiré en termes d'économie narrative, puisque, selon lui, jusqu'à $20 \%$ des épisodes diffusés hebdomadairement sont consacrés au récapitulatif ou au teaser ${ }^{18}$. La narration continue permet également de ménager des arcs narratifs plus denses et d'envisager le déroulement de la mise en intrigue sur une longue période. Ainsi que l'a mis en évidence Marjolaine Boutet (2015), l'importance du long terme est problématisée dès le premier épisode. Frank Underwood se voit privé du poste de Secrétaire d'État que lui avait promis Garrett Walker (Michel Gill), fraîchement élu président, en échange de son soutien. Il planifie dès lors une vengeance sur le très long terme avec l'aide de sa femme, comme en témoigne ce dialogue avec son directeur de cabinet et âme damnée, Doug Stamper (Michael Kelly), extrait du chapitre 1 :

FRANK : Tous. Je les tiens tous pour responsables.

DOUG : Vengeance?

FRANK : Non! Plus que ça. Prenez un peu de recul. Considérez la situation dans son ensemble. 
DOUG : Je crois que je vois ou vous voulez en venir. Kern d'abord?

FRANK : C'est comme ça qu'on dévore une baleine, Doug. Une bouchée à la fois.

Boutet voit dans ce passage une illustration de l'«approche globale du temps » que manifeste Underwood :

L'approche globale du temps de Frank Underwood apparaît clairement dans ce dialogue : il va mettre patiemment son plan en marche (" une bouchée à la fois »), en faisant d'abord avancer prudemment ses pions sur l'échiquier, mais en accélérant à la fin lorsqu'il se rapprochera de l'échec et mat ${ }^{19}$. (Boutet $2015: 86$ )

Son analyse concerne le niveau diégétique (séquence actionnelle), mais la conception du personnage ne doit pas être découplée de la mise en intrigue, dont elle est solidaire. En effet, la préparation d'un plan de vengeance complexe par le protagoniste conditionne les attentes des spectateurs, qui savent d'emblée qu'ils n'auront accès au dénouement qu'après de nombreuses heures de visionnage et plusieurs années de patience. Dès les premières scènes du premier épisode, la série met en place un dispositif métaleptique: Frank Underwood s'adresse directement au spectateur à travers de fréquents apartés dans lesquels il détaille ses plans avec cynisme. Suivant Mario Klarer, Frank apparaît donc comme « l'équivalent d'une figure d'auteur qui est, paradoxalement, responsable de l'organisation de l'intrigue depuis l'intérieur de l'intrigue $^{20} »$ (Klarer $2014: 213$ ). En cohérence avec cette idée, on peut observer une "diégétisation» (Odin 2000) du chapitrage. Davantage que l'indication d'un découpage, les mentions «Chapitre 1 », «Chapitre 2 », etc. constituent des titres, ce qui explique le redoublement entre les numérotations épisodique et chapitrale dans le menu de la série sur le site de Netflix. Signalons en outre que la numérotation des chapitres de House of Cards se distingue de la numérotation habituelle des épisodes de série, puisque le décompte ne recommence pas à chaque nouvelle saison : le premier épisode de la saison 2 est intitulé « Chapitre 14 », le premier de la saison 3, «Chapitre 27 », etc. En conséquence, l'autonomie épisodique est atténuée au profit d'une économie narrative qui privilégie une continuité surplombante. De plus, le chapitrage n'apparaît que dans le menu et n'est signalé par aucun intertitre au sein de l'épisode. Ce lissage des seuils saisonniers accentue encore l'effet de continuité au sein d'un tout et la dimension épique du récit. Il est redoublé diégétiquement par des effets de continuité chronologique parfois très prononcés. Ainsi, à la fin du premier épisode, Frank, installé à la terrasse de son restaurant préféré, en train de dévorer des côtes de porc, prononce une phrase à double sens, qui s'adresse à la fois au propriétaire du restaurant et au spectateur, auquel il jette un coup d'œil face caméra : "Je suis affamé aujourd'hui. » L'épisode suivant reprend immédiatement là où le précédent s'était arrêté : Frank, toujours assis à la même table, s'essuie la bouche à la fin de son repas. Cette stratégie est reprise entre la fin de la première saison et le début de la deuxième, dans lequel Frank et Claire poursuivent le jogging qu'ils avaient commencé avant l'hiatus saisonnier, ce qui souligne le décalage entre la temporalité diégétique et la temporalité de la réception.

Ensuite, le découpage chapitral, contrairement au découpage épisodique, n'est pas lié à ce que l'on peut identifier comme les temps forts de la mise en intrigue, aux pics de tension narrative, et Willimon marque nettement ses distances avec les stratégies de découpage du feuilleton, notamment avec le cliffhanger :

Je pouvais instaurer quelque chose au début de la saison qui ne reviendrait pas avant la fin de la saison deux. Cela signifie un récit plus stratifié et plus sophistiqué. Nous espérions que les gens voudraient regarder immédiatement l'épisode suivant, non pas grâce à un quelconque cliffhanger superficiel, mais grâce à leur 
investissement dans une histoire et des personnages complexes; c'est cela qui les fait revenir ${ }^{21}$.

Ainsi, les scènes les plus marquantes n'ont pas lieu à la fin des épisodes, mais souvent dix à quinze minutes avant, à l'instar des assassinats de Peter Russo ("Chapitre 11 ", S01E11) et de Zoe Barnes («Chapitre $14 »$, S02E01) par Frank Underwood, de Rachel Posner par Doug Stamper («Chapitre 39», S03E13) et de Tom Yates par Claire Underwood («Chapitre 64 », S05E09). L'idée que le découpage feuilletonesque serait superficiel, artificiel, suppose qu'il existe à l'inverse des découpages plus «naturels». Cette conception n'est pas si éloignée de la recherche de "naturalisation» du chapitrage dans le roman naturaliste, qui, pour citer Philippe Hamon, «se présente comme un "pré-découpage" du référent, de la réalité physique ou sociale elle-même " (2017: 12), comme s'il calquait de manière transparente la temporalité du monde raconté, indépendamment de toute mise en récit, et donc comme s'il ne résultait pas d'une organisation séquentielle. De fait, on retrouve dans House of Cards un mode de découpage fréquent dans le roman : les changements de chapitre peuvent par exemple coïncider avec les déplacements des personnages. Par exemple, dans le "Chapitre 8 ", Frank Underwood retourne dans son ancienne université, tandis que Peter Russo se rend dans sa ville natale; le "Chapitre 60 » correspond à un déplacement de Frank dans l'Ohio, afin de participer à un rassemblement secret qui réunit les hommes les plus puissants du pays. Ainsi, les potentialités esthétiques de la suspension sont mises en sourdine, au profit de la seule fonction respiratoire du découpage, qui sert avant tout à ménager des lieux où l'on peut aisément (c'est-à-dire sans trop d'effort cognitif) quitter ou reprendre la progression dans un récit trop long pour être actualisé en une seule session.

\section{Une prise de pouvoir des spectateurs?}

12 L'usage du chapitrage dans House of Cards est l'un des éléments qui témoignent d'une volonté de mettre fin au règne de l'épisode comme unité narrative normative. On assiste à une dilution de l'effet de seuil, qui devient optionnel, contrairement à ce qui se passe dans le cadre de la diffusion télévisuelle. Il s'agit alors d'un seuil " discret », qui suggère aux spectateurs d'éventuelles pauses, sans pour autant se faire prescriptif. Willimon insiste sur l'adaptabilité du mode de diffusion aux différentes pratiques, puisque, comme dans un roman, le spectateur peut ménager ses pauses quand il le souhaite :

Beaucoup de gens ne «binge-watchent " pas. C'est en cela que la situation est à nouveau similaire au roman : si quelqu'un sort un roman de 600 pages, il y aura des gens qui liront tout d'un coup, et d'autres qui mettront plusieurs années à le lire. Il faut que cela fonctionne des deux manières. La chose la plus importante est l'émancipation du public. Vous donnez au public - particulièrement avec la sortie immédiate d'une saison complète - la possibilité de choisir son expérience. S'il a le choix, il y a plus de chances qu'il reçoive le récit d'une manière proche de celle que vous voulez ${ }^{22}$.

Donc, paradoxalement, dans ce discours téléologique, l'aboutissement des séries feuilletonnantes est conçu comme la suppression des spécificités de la narration sérielle. Ainsi Jason Mittell peut-il légitimement se demander si «ces récits “multiépisodes" peuvent encore être considérés comme feuilletonnants ${ }^{23}$ " (2015: 41). L'idéal de la disparition complète du seuil épisodique apparaît nettement dans le 
discours de Willimon, qui rêve d'écrire une série où seule persisterait la rupture saisonnière :

J'ai une idée pour mon prochain projet: une saison de six heures en flux. Sans pause. Sans épisode. Vous mettez en pause quand vous voulez, ou pas du tout. À ce niveau, qu'est-ce que c'est? Une série télévisée, une saison ou un épisode ? Qui le sait et qui s'en soucie finalement? Nous avons toujours parlé de House of Cards comme d'un film; nous avons conçu la première saison comme un film de 13 heures. Nous ne faisons pas d'épisodes avec un début, un milieu et une fin qui ont une existence autonome. La raison pour laquelle les épisodes ne sont pas titrés - nous les appelons des « chapitres » - est qu'ils n'ont pas besoin d'être pleinement résolus. Dans le bureau des scénaristes, nous ne parlons pas d'une histoire A ou d'une histoire $\mathrm{B}$, ou d'autres choses caricaturales qu'on trouve dans cette horrible section de Barnes \& Noble qui vous explique soi-disant comment écrire un scénario ${ }^{24}$.

Cet idéal pose toutefois certains problèmes si on l'envisage du côté de la réception. Revenons un instant sur une phrase de Willimon que j'ai évoquée plus haut: «Je pouvais instaurer quelque chose au début de la saison qui ne reviendrait pas avant la fin de la saison deux. » Transparaît ici la volonté des créateurs d'une maîtrise absolue du rythme narratif: rien n'est arbitraire, tout est planifié, ou du moins, des pistes narratives potentielles sont ouvertes en permanence, qui pourront ou non être utilisées ultérieurement. En conséquence, la réception de House of Cards suppose une densification de l'activité anticipatrice : tout élément qui apparaît à l'écran peut avoir des implications sur le long terme, ce qui encourage le spectateur à l'envisager comme l'indice d'éventuels scénarios possibles. Le spectateur idéal que tentent de programmer les créateurs et les producteurs de ce genre de récit est donc " hyper-compétent », en quelque sorte. Il est capable d'une compréhension et d'une mémorisation exhaustive de tous les événements qui se sont déroulés au sein du monde narratif. Il est capable de stocker mentalement tous les détails qui peuvent sembler anodins, dans la mesure où ils sont susceptibles d'être exploités par la mise en intrigue des dizaines d'épisodes plus tard, et de les réactiver dans les moments pertinents afin de produire une interprétation correcte ${ }^{25}$. Dans la pratique, ce mode de réception se révèle assez compliqué à mettre en place pour les spectateurs réels : les saisons étant livrées à un an d'intervalle, il est quasiment impossible d'avoir en mémoire toutes les informations utiles, à moins de visionner à nouveau l'intégralité des saisons précédentes avant chaque nouvelle saison ou d'en lire des résumés exhaustifs. Ces résumés sont toujours accessibles sur Wikipédia, et mis à jour très rapidement, ce qui est un signe de la réactivité des fans, ou de la participation des producteurs à cette activité. C'est ici que l'analogie avec le modèle romanesque trouve ses limites : autant un lecteur qui a besoin de se rafraîchir la mémoire peut le faire sans trop de difficulté en retournant quelques pages en arrière, autant la manœuvre est beaucoup plus complexe et décourageante dans le cas des plateformes de VàD. Par ailleurs, le chapitrage se superpose avec le balisage familier du public dans le menu de Netflix: il y a ici coexistence du paradispositif et du dispositif, qui habituellement se succèdent dans le temps, ce qui montre bien qu'on ne se débarrasse pas si facilement des modèles de diffusion sériels classiques.

En outre, on se rend compte que l'hiatus saisonnier n'est jamais remis en cause, il est au contraire revendiqué comme un seuil légitime. L'effet de seuil est donc concentré sur la saison et il est frappant de constater qu'on retrouve dans les dernières scènes des saisons les stratégies habituelles de relance narrative du feuilleton, comme des scènes choquantes ou des retournements de situation qui débouchent sur un effet de 
cliffhanger, pourtant dénoncés comme artificiels. Ainsi la troisième saison se clôt sur une scène qui ne dépareillerait pas dans un soap opera: dans la dernière réplique de l'épisode, Claire annonce à Frank qu'elle le quitte. Dans la dernière scène de la quatrième saison, les Underwood assistent sans ciller à la décapitation d'un otage. Et à la fin de la cinquième saison - pour laquelle Melissa James Gibson et Frank Pugliese assurent le rôle de showrunners, suite au départ de Beau Willimon-, Claire décide de se retourner contre son mari et de faire cavalier seul, bref, de ne plus être réduite au rôle de second couteau. Elle s'adresse directement au spectateur, mode d'énonciation jusqu'alors exclusivement réservé à Frank, et déclare pour finir, face à la caméra : «À mon tour ", ce qui revient à affirmer un nouveau rôle de protagoniste - voire d'auteure de sa propre histoire, au même titre que l'était précédemment Frank. Ces renégociations de la relation de couple, et des enjeux de pouvoirs que cela implique, sont évidemment situées à un seuil stratégique : le spectateur est amené à anticiper une dynamique narrative antithétique à la saison précédente, ce qui augmente son impatience d'accéder à la saison suivante et assure sa fidélisation.

14 On retrouve donc des problématiques habituelles dans le domaine de la sérialité, seule l'échelle change, les charnières de la sérialité se déployant désormais sur des enjeux macro-narratifs. Si House of Cards demeure sérielle, c'est donc surtout au niveau de l'enchaînement de ses saisons, qui n'est pas totalement planifié, et dont la diffusion reste segmentée et soumise aux réactions du public-ainsi qu'en témoigne la disparition soudaine de Frank Underwood à la fin de la sixième saison, suite aux accusations de harcèlement dont a fait l'objet Kevin Spacey ${ }^{26}$.

\section{Le chapitrage comme stratégie de branding}

Dans le cas de House of Cards, les créateurs ont largement théorisé la question du format ${ }^{27}$ et des usages. Il s'agit très clairement d'une stratégie de distinction : l'intitulé marque une prise de distance par rapport au modèle épisodique, une volonté d'innovation narrative, tout en se situant dans une tradition prestigieuse. Le chapitrage est doté d'une fonction revendicatrice et marque l'affirmation d'une rupture avec un modèle préexistant. Cette émancipation du support télévisuel se situe dans la lignée d'une stratégie de communication inaugurée par HBO, dont le slogan est désormais bien connu: "It's not TV, it's $H B O$ ». Elle repose sur la valorisation de l'autonomie auctoriale, considérée comme synonyme de projets innovants et originaux. Le discours téléologique des instances productrices, qui dévalorise les autres modes de production en insistant notamment sur la contrainte artificielle que constituerait l'épisode, s'inscrit nettement dans cette stratégie. Le chapitrage signale un double «empowerment »: la prise de pouvoir des créateurs, qui sont présentés comme l'autorité régissante unique, débarrassée des contraintes de production et de celles relatives au support médiatique; et la prise de pouvoir des spectateurs, qui peuvent gérer l'actualisation à leur guise ${ }^{28}$. Dans une conférence tenue en 2013, Spacey défendait ainsi la pertinence économique d'un modèle fondé sur la liberté des usagers :

Clairement, le succès du modèle Netflix, qui diffuse une saison entière de House of Cards en une seule fois, a prouvé une chose : le public veut le contrôle. Il veut la

liberté. S'il veut "binger ", comme il l'a fait pour House of Cards et pour beaucoup

d'autres séries, alors on doit le laisser « binger $»^{29}$.

On assiste à une normalisation du "binge-watching", qui est intégré au dispositif de la plateforme comme une norme de visionnage, voire comme le mode de consommation 
préférentiel, et qui n'est plus décrit comme une dérive addictive, ainsi que le note McCormick :

[L]orsqu'on envisage la différence entre le binge-watching d'un DVD ${ }^{30}$ et le bingewatching d'une plateforme de VàD, il faut analyser la manière dont les différentes interfaces encouragent (ou parfois empêchent) ce que j'appelle du binge-watching assoupli. [...] Avec les interfaces de DVD qui n'incluent pas le bouton «lire l'intégralité ", le binge-watching est marqué par des pauses qui exigent de naviguer sur le menu pour aller à l'épisode suivant. En revanche, les plateformes de VàD telles que Netflix utilisent souvent une structure en lecture automatique par défaut, ce qui implique une action minimale de la part de l'utilisateur pour continuer à « binger $»^{31}$. (McCormick $2016: 103$ )

Dans le même sens, selon Mareike Jenner, le dispositif de Netflix est entièrement structuré par le «binge-watching» (2018: 109-118). Le chapitrage de House of Cars est ainsi l'un des nombreux phénomènes marquant l'avènement d'une "culture binge ", selon le terme de McCormick (2016 : 103), encouragée par les créateurs qui révèlent s'y adonner eux-mêmes :

Je peux vous en parler d'expérience parce que pour la première fois, il y a deux semaines, Beau, Josh Donen, Eric Roth et moi avons regardé ensemble les 13 heures du début à la fin. Et c'est fou. C'est comme un livre. C'est comme si vous lisiez un chapitre, arrêtiez la lecture pour acheter du thaï, reveniez, et la relanciez. Ça fonctionne différemment. Le rythme de consommation informe d'une certaine manière le type de relation que vous avez avec les personnages, qui est très différent de la télévision traditionnelle ${ }^{32}$.

Ce que McCormick désigne comme une "culture binge ", c'est aussi un renversement des représentations, une stratégie de légitimation de cette pratique qui la rapproche d'une forme d'élitisme, de la culture "highbrow». Il ne s'agit pas seulement de déculpabiliser les spectateurs, mais de faire du «binge-watching " une activité culturelle à part entière, dont on peut tirer du prestige symbolique. Selon l'idéologie que les producteurs cherchent à établir, le spectateur qui a regardé tous les épisodes d'affilée n'a pas perdu un temps précieux, qu'il aurait pu consacrer à des activités plus constructives, il s'est livré à la réception d'un récit aussi complexe, aussi dense et aussi stimulant qu'un roman de Tolstoï :

La conception positive de l'immersion narrative par Sarando suggère une ontologie

du contenu textuel qui s'éloigne des pauses et des hiatus pour privilégier une

expérience textuelle plus cohérente ${ }^{33}$. (McCormick 2016 : 102)

On en revient ici à une conception traditionnelle de l'immersion fictionnelle, qui serait limitée au moment de l'actualisation, sans inclure les moments de réactivation imaginative qu'encourage la sérialité. Le degré d'immersion serait ainsi proportionnel à l'ampleur et à la densité du récit, qui supposerait symétriquement un monde narratif riche et complexe. Il dépendrait également de la continuité dans le temps de la réception: le modèle de diffusion épisodique est remis en cause par les producteurs, parce qu'il suppose selon eux une expérience moins immersive. Par conséquent, Netflix ne vend pas seulement des séries, mais aussi une représentation acceptable d'un mode de consommation. Or, le "binge-watching" demeure un mode de consommation minoritaire d'après Mittell, puisque la sortie d'une saison « a une grande incidence sur la conscience du public sur le moment, mais diminue très rapidement ensuite ${ }^{34}$ » et que le nombre de spectateurs de House of Cards n'est pas, selon lui, à la hauteur de son influence médiatique. L'exagération de la valeur révolutionnaire du format de House of Cards relève donc du branding de Netflix. Comme le remarquait Mark Lawson, journaliste du Guardian, House of Cards s'avère finalement «moins innovante dans son récit que dans sa diffusion ${ }^{35} »$. 
Le chapitrage des séries télévisées ne se réduit donc pas à des enjeux poétiques et esthétiques, il ne se comprend pas indépendamment du contexte médiatique qui l'englobe. Comme je l'ai souligné en introduction, il implique une grande variété d'intentionnalités, de modes de réception, et ne se réduit pas à un usage fixe, dans la mesure où il ne correspond pas à la terminologie conventionnelle du découpage sériel. Dans le cas de House of Cards, l'affichage d'une segmentation en chapitres va également de pair avec une valorisation de l'autonomie des créateurs. Elle est déterminée par des enjeux économiques qui dépassent les séries elles-mêmes : il s'agit non seulement de s'adapter à la demande, mais d'anticiper les comportements des usagers, de leur donner ce qu'ils veulent, mais avant qu'ils sachent qu'ils le veulent. Dans ce contexte, le discours qui souligne l'«empowerment» $d u$ public s'avère paradoxal. Les problématiques de la sérialité narrative que j'ai mises en avant jusqu'à présent (adaptation aux contraintes externes, insertion dans la temporalité médiatique, etc.) ne sont pas pour autant évacuées : seule leur échelle est modifiée. Ainsi, la libération de la dépendance de la segmentation du récit sériel envers l'organisation de son véhicule médiatique est plus marquée avec la VàD, en particulier quand certaines chaînes en streaming proposent la diffusion immédiate d'une saison complète. Mais si les plateformes de VàD remettent en cause les seuils épisodiques, les logiques de production et de réception demeurent profondément sérielles. Un certain nombre de traits définitoires de la narration sérielle restent donc pertinents, ne serait-ce qu'à l'échelle de la saison. Alors que le chapitrage de House of Cards fait généralement office de pause respiratoire, les fins de saisons s'achèvent sur des cliffhangers, ce qui traduit la persistante des mécanismes feuilletonnants. Raphaël Baroni et moi-même avions souligné l'ambition des productions télévisuelles actuelles, qui cherchent à faire coïncider des moyens de production importants avec les valeurs constitutives du souschamp de la production restreinte, combinant ainsi un pragmatisme économique avec ce que l'on pourrait appeler un "pragmatisme symbolique» (Baroni et Goudmand 2017). House of Cards correspond à ces nouveaux enjeux, dans la mesure où la série n'est pas immédiatement rentable économiquement, mais où elle rapporte un capital symbolique à la chaîne et lui permet d'attirer de nouveaux abonnés. Mittell l'envisage ainsi comme un "loss leader»: elle fait perdre de l'argent à Netflix, mais elle est " conçue pour faire de la publicité à la chaîne et attirer des abonnés dans le cadre d'une stratégie sur le long terme ${ }^{36} »$. Rappelons qu'il est difficile de se prononcer avec précision sur la rentabilité d'une série diffusée par Netflix, qui ne rend pas publics ses chiffres d'audience. Toutefois, une estimation a été faite en 2015 par la compagnie Luth Research à partir d'un sondage auprès de 2500 abonnés américains, d'après laquelle 2,66 millions de spectateurs auraient regardé la saison 3 de House of Cards dans les 30 jours suivant sa sortie; elle est battue par deux nouvelles séries, Daredevil (Netflix 2015-) et Unbreakable Kimmy Schmidt (Netflix 2015-)37. Ceci confirme l'hypothèse de Mittell selon laquelle le rôle essentiel de House of Cards est celui d'une vitrine : les séries les plus «marquantes » - c'est-à-dire celles qui sont mises en avant par les chaînes et distinguées par les critiques au sein d'une production pléthorique - ne sont donc pas forcément les plus regardées. En 2016, avec des audiences estimées à 5,67 millions dans le monde entier - chiffre considéré comme assez médiocre par l'industrie télévisuelle elle n'est que la neuvième série la plus regardée de la chaîne ${ }^{38}$. Malgré la fonction prototypique qui lui a été attribuée dans les discours promotionnels, le modèle proposé par House of Cards n'a pas véritablement fait cas d'école. Le chapitrage n'est pas devenu une nouvelle norme, il apparaît comme une exception plutôt qu'une règle, que ce soit 
sur Netflix ou sur les autres plateformes qui produisent du contenu original, telles que Amazon et Hulu: un certain nombre de séries Netflix ont certes adopté depuis un découpage en chapitres, telles que The OA (2016-), Stranger Things (2016-), Dear White People (2017-), The Chilling Adventures of Sabrina (2018-), The Kominsky Method (2018-), pour n'en citer que quelques-unes, mais ce choix reste minoritaire parmi les contenus originaux de la plateforme. Il n'y a pas eu plus de glissement terminologique qui marquerait le passage des épisodes livrés de façon hebdomadaire aux épisodes rassemblés au sein d'un tout, qu'il s'agisse d'un DVD ou d'une plateforme de VàD, et le terme d'épisode continue d'apparaître comme suffisamment souple et consensuel pour inclure le découpage de séries hyper-feuilletonnantes. On observe désormais ponctuellement l'adoption d'un chapitrage par des séries récentes diffusées hebdomadairement, à l'instar de The Mandalorian (2019-), première série originale diffusée sur la plateforme Disney+. Disney+, comme c'était déjà le cas de Hulu, a donc opté pour un rythme diffusion qui renoue avec les pratiques hertziennes, en raison notamment des avantages financiers qu'il présente ${ }^{39}$, ce qui marque une évolution dans les stratégies des plateformes de VàD. Cet infléchissement s'observe d'ailleurs également du côté de Netflix, qui tend depuis quelques années à recalibrer son discours autour de la notion de diversité ${ }^{40}$, au détriment de celle de "binge-watching " (Jenner $2018: 161-182)$.

\section{BIBLIOGRAPHIE}

Baroni, Raphaël et Goudmand, Anaïs, 2017, « Le feuilleton littéraire et télévisuel : du stéréotype à l'approfondissement du personnage ", dans J. Cléder et $\mathrm{F}$. Wagner (dir.), Le cinéma de la littérature, Lormont, Éditions Nouvelles Cécile Defaut, p. 187-203.

Bénassi, Stéphane, 2000, Séries et feuilletons TV : pour une typologie des genres fictionnels, Liège, CEFAL.

Boutet, Marjolaine, 2015, « The Politics of Time in House of Cards », dans B. Brigit, A. Ganser et N. Poppenhagen (dir.), Transgressive Television: Politics, Crime and Citizenship in 21st-century American TV Series, Heidelberg, Universitätsverlag Winter, coll. « American Studies Monograph Series », p. 83-102.

Campion, Baptiste, 2019, « Regarder des séries sur Netflix : l'illusion d'une expérience spectatorielle augmentée », TV Series, ${ }^{\circ}{ }^{\circ}$ 15, [En ligne], DOI : 10.4000/tvseries.3479.

Combes, Clément, 2013, La pratique des séries télévisées : une sociologie de l'activité spectatorielle, thèse de doctorat, Paris, Mines ParisTech.

Combes, Clément, 2015, « Du rendez-vous télé au binge watching : typologie des pratiques de visionnage de séries télé à l'ère numérique ", Études de communication, n 44, p. 97-114, [En ligne], DOI : 10.4000/edc.6294.

Cornillon, Claire, 2018, Sérialité et transmédialité. Infinis des fictions contemporaines, Paris, Honoré Champion. 
Dionne, Ugo, 2008, La voie aux chapitres : poétique de la disposition romanesque, Paris, Seuil, coll. « Poétique ».

Glebatis Perks, Lisa, 2014, Media Marathoning: Immersions in Morality, Londres, Lexington Books. Hamon, Philippe, 2017, « Ouverture », dans C. Colin, T. Conrad et A. Leblond (dir.), Pratiques et poétiques du chapitre du XIX ${ }^{e}$ au XXI ${ }^{e}$ siècle, Rennes, PUR, coll. «Interférences », p. 7-18.

Hills, Matt, 2005, « Patterns of Surprise: The "Aleatory Object” in Psychoanalytic Ethnography and Cyclical Fandom », American Behavioral Psychologist, vol. 48, n 7, p. 801-821.

Hills, Matt, 2007, « From the Box in the Corner to the Box Set on the Shelf: "TVIII" and the cultural/textual valorisations of DVD », New Review of Film and Television Studies, n 5, p. 41-60.

Jenkins, Henry, [2006] 2013, La culture de la convergence : des médias au transmédia, trad. Christophe Jaquet, Paris, Armand Colin, coll. « Médiacultures ».

Jenner, Mareike, 2015, « Binge-watching: Video-on-demand, quality TV and mainstreaming fandom », International Journal of Cultural Studies, vol. 20, n 3, p. 304-320.

Jenner, Mareike, 2018, Netflix and the Re-invention of Television, New York, Springer International Publishing AG.

Klarer, Mario, 2014, «Putting television “aside”: novel narration in House of Cards », New Review of Film and Television Studies, vol. 12, $\mathrm{n}^{\circ}$ 2, p. 203-220.

Marshall, Philip David, 2009, « Screens: Television's Dispersed "broadcast” ", dans G. Turner et J. Tay (dir.), Television Studies After TV: Understanding Television in the Post-Broadcast Era, Londres, New York, Routledge, p. 41-50.

McCormick, Casey, 2016, " "Forward is the Battle Cry”: Binge-Viewing Netflix's House of Cards ", dans K. McDonald et D. Smith-Rowsey (dir.), The Netflix Effect: Technology and Entertainment in the 21st Century, New York, Bloomsbury Academic, p. 101-116.

Mittell, Jason, 2015, Complex TV: the Poetics of Contemporary Television Storytelling, New York, Londres, New York University Press.

Monnet-Cantagrel, Hélène, 2018, Le format bible des séries télévisées. Pratique et enjeux esthétiques, Paris, L'Harmattan.

Odin, Roger, 2000, De la fiction, Paris, Bruxelles, De Boeck Université, coll. « Arts \& cinéma ».

Pichard, Alexis, 2011, Le nouvel âge d'or des séries américaines, Paris, Le Manuscrit.

Thompson, Robert J., 1997, Television's Second Golden Age: From Hill Street Blues to ER, Syracuse, NY, Syracuse University Press, coll. « The Television Series ».

\section{NOTES}

1. Ce découpage, adventice du point de vue du visionnage de l'épisode en DVD, n'est cependant pas arbitraire puisqu'il respecte la logique narrative et qu'il reprend les seuils correspondant aux coupures publicitaires de la diffusion initiale (si elles existent).

2. BBC, 2005-.

3. HBO, 2011-2019.

4. AMC, 2008-2013. 
5. The WB, UPN 1997-2003.

6. " paternalistic form of delivery ».

7. Le terme est dérivé de l'expression «binge-drinking», qui désigne une pratique consistant à boire de grandes quantités d'alcool en un temps très court. Cette « perte de contrôle » est toutefois paradoxale, puisqu'elle découle justement d'un contrôle accru, d'une émancipation des contraintes de visionnage, autrefois fixées par des dispositifs médiatiques dépourvus de possibilités d'interaction avec le contenu. Une autre terminologie, héritée du lexique fanique des années 1980, est parfois préférée par les critiques afin d'éviter les connotations péjoratives de la précédente, celle du «marathon» (Glebatis Perks 2014). Les séances de «marathon» sont en effet antérieures au développement des plateformes de streaming: les fans, profitant de l'éditorialisation des séries (VHS, puis DVD, puis téléchargement...), en organisaient eux-mêmes, et elles étaient parfois même orchestrées par les chaînes, à l'instar des marathons 24 heures chrono organisés par la Fox puis par Canal+ (voir Combes 2013 : 168-169).

8. Beau Willimon, cité par Nathan Mattise, 2 janvier 2013, « House of Cards: The "13-hour movie" defining the Netflix experience ", Ars Technica, [En ligne], https:// arstechnica.com/information-technology/2013/02/house-of-cards-the-13-hour-moviedefining-the-netflix-experience/. Je traduis toutes les citations en anglais.

9. Ted Sarandos, cité par David Carr, 24 février 2013, "Giving Viewers What They Want ", The New York Times, [En ligne], https://www.nytimes.com/2013/02/25/ business/media/for-house-of-cards-using-big-data-to-guarantee-its-popularity.html.

10. Voir « Do You Know When You Were Hooked? Netflix Does », Netflix Media Center, 23 septembre 2015, [En ligne], https://media.netflix.com/en/press-releases/do-youknow-when-you-were-hooked-netflix-does.

11. Beau Willimon, cité par Andrew Romano, 15 mai 2013, «Why you're addicted to TV », Newsweek, [En ligne], http://www.newsweek.com/2013/05/15/why-youreaddicted-tv-237340.html.

12. Kevin Spacey, 23 août 2013, Conference MacTaggart, Edinburgh International Television Festival, conférence citée.

13. Vince Gilligan, cité par Jon Bilstein, 7 janvier 2014, «Vince Gilligan Talks Favorite TV Technologies at CES », Rolling Stone, [En ligne], https://www.rollingstone.com/tv/ news/vince-gilligan-talks-favorite-tv-technologies-at-ces-20140107.

14. La notion d' "âge d'or » des séries télévisées, souvent définie suivant des critères normatifs, et sa périodisation ne font pas consensus parmi les chercheurs : pour Robert J. Thompson (1997), un second âge d'or débute en 1980, succédant au premier des années 1950; pour Alexis Pichard (2011), le second âge d'or débute dans les années 2000, les productions des années 1980 n'en constituant que les prémisses.

15. Beau Willimon, cité par Emily Buder, 15 septembre 2014, «Beau Willimon on "House of Cards", Bad Television and His "Crazy" Next Project », IndieWire, [En ligne], http://www.indiewire.com/2014/09/beau-willimon-on-house-of-cards-bad-televisionand-his-crazy-next-project-22198/.

16. Beau Willimon, cité par Alison Willmore, 29 janvier 2013, " "House of Cards" Showrunner Beau Willimon on Netflix, Ideal Politicians and Working With David Fincher ", IndieWire, [En ligne], http://www.indiewire.com/2013/01/house-of-cards- 
showrunner-beau-willimon-on-netflix-ideal-politicians-and-working-with-davidfincher-41572/.

17. Trilogie « Francis Urquhart »: House of Cards (1989), The Play of King (1992), The Final Cut (1994).

18. Ted Sarandos, cité par Andrew Romano, 15 mai 2013, art. cit. Selon Baptiste Campion, ce lissage des seuils épisodiques se situe en fait dans la continuité des pratiques des networks, qui tendaient à diffuser les crédits de fin en vitesse accélérée dans une fenêtre miniature de l'écran, diffusée en parallèle d'une annonce publicitaire (2019 : §28).

19. «It is clear in this dialogue that Frank Underwood has a comprehensive approach to time: he will patiently set his plan in motion ("one bite at a time"), at first moving his pieces on the chessboard carefully, but accelerating in the end when checkmate is near."

20. « an author-like figure who is, paradoxically, responsible for orchestrating the plot from within the plot. »

21. Beau Willimon, cité par Nathan Mattise, 2 janvier 2013, « House of Cards: The "13hour movie" defining the Netflix experience", Ars Technica, [En ligne], https:// arstechnica.com/information-technology/2013/02/house-of-cards-the-13-hour-moviedefining-the-netflix-experience/.

22. Beau Willimon, cité par Emily Buder, 15 septembre 2014, op. cit.

23. « ... whether these multiepisode narratives can be considered serial at all. »

24. Ibid.

25. Une telle gestion de l'économie narrative n'est pas inédite dans les séries télévisées: Robert J. Thompson (1996: 75-98) montre ainsi que les scénaristes de St. Elsewhere (NBC, 1982-1988) multiplient les références intra- et intertextuelles, ce qui peut notamment s'expliquer par le développement de la télécommande, qui permet aux téléspectateurs de naviguer à leur guise dans les contenus.

26. Le scandale sexuel impliquant Spacey a contraint les scénaristes à écrire la septième et ultime saison sans son protagoniste. La production de la saison a dès lors été interrompue le 30 octobre 2017, alors qu'elle venait de commencer, avant de reprendre fin janvier 2018, pour huit épisodes au lieu des treize habituels. Les scénaristes ont donc dû complètement remanier l'intrigue et trouver des motivations diégétiques à l'absence de Frank Underwood.

27. J'emploie ici le terme "format» dans le sens où l'entend notamment Stéphane Bénassi (« le format d'un épisode résulte de la triple conjonction de sa forme syntaxique, de sa stratégie de programmation et de sa durée ", $2000: 32$ ), et non dans le sens de «bible» pour désigner le document que fournissent les créateurs de séries pour présenter le cadre destiné à se répéter dans chaque épisode, comme dans les travaux de Hélène Monnet-Cantagrel (2018:32).

28. Mareike Jenner montre que l'idéologie néolibérale sous-jacente à ce type de discours est historiquement située: la valorisation de la prise de pouvoir du consommateur justifiait déjà le développement de la télécommande et du magnétoscope (2018: 35-45).

29. Kevin Spacey, 23 août 2013, Conference MacTaggart, Edinburgh International Television Festival. La conférence est visible dans son intégralité sur le site du Guardian 
: https://www.theguardian.com/media/video/2013/aug/23/kevin-spacey-mactaggartlecture-video.

30. Sur le «binge-watching » de séries en DVD, voir également Hills (2005 et 2007).

31. « in considering the difference between binging on a DVD and binging on a SVOD platform, we should analyze how different interfaces encourage (or sometimes hinder) what I call "smooth binging" experiences. [...] With DVD interfaces that do not include the "play all" button, binge-viewing is marked by pauses that require menu navigation to get to the next episode. On the other hand, SVOD interfaces like Netflix usually default to an "autoplay" structure, in which limited user action is needed to continue the binge. »

32. David Fincher, cité par Alan Sepinwall, 29 janvier 2013, « "House of Cards" director David Fincher on making 13 hours for Netflix », Uproxx, [En ligne], https://uproxx.com/ sepinwall/house-of-cards-director-david-fincher-on-making-13-hours-for-netflix/.

33. «Sarando's positive framing of narrative immersion suggests an ontology of textual content that moves away from pauses and hiatuses toward a more cohesive textual experience. »

34. Jason Mittell, cité par Sally Pollack, 13 mars 2015, « Congressman Welch, too, binges on House of Cards", Burlington Free Press, [En ligne], https:// www.burlingtonfreepress.com/story/news/local/2015/03/13/welch-america-binginghouse-cards/70276280/.

35. Mark Lawson, 22 février 2015, "House of Cards review: less innovative in its narrative than in its distribution", The Guardian, [En ligne], https:// www.theguardian.com/tv-and-radio/2015/feb/27/house-of-cards-series-threepresident-francis-underwood-kevin-spacey-robin-wright.

36. Jason Mittell, cité par Sally Pollack, 13 mars 2015, op. cit.

37. Steve Baron, 28 avril 2015, « Research Firm Measures "Ratings" for Netflix Original Series; "Daredevil" is Most-Watched ", Tv By The Numbers, [Indisponible], https:// web.archive.org/web/20191105133935/https://tvbythenumbers.zap2it.com/ uncategorized/research-firm-measures-ratings-for-netflix-original-series-daredevil-ismost-watched/395824/ (archive).

38. Aïssatou Loum, 26 août 2016, «Stranger Things : troisième série la plus regardée de Netflix ", Brain Damaged, [En ligne], http://braindamaged.fr/26/08/2016/strangerthings-troisieme-serie-la-plus-regardee-de-netflix/.

39. Pour voir la saison complète de The Mandalorian, qui comprend huit épisodes, un abonné doit payer deux mois au lieu d'un seul comme si toute la saison avait été livrée le même jour.

40. En mettant en avant le caractère international de la production et de la réception des contenus originaux. 


\section{RÉSUMÉS}

Cet article vise à analyser l'usage du chapitrage dans une série produite par Netflix, House of Cards (2013-2018). Il s'agit d'interroger le lien entre le recours à la notion de chapitre et le contexte de diffusion de la série, via une plateforme de streaming qui livre l'intégralité des épisodes d'une saison le même jour, abandonnant ainsi le hiatus hebdomadaire : le public est alors libre de choisir son rythme d'actualisation, comme dans le cas d'un roman chapitré. On cherchera à déterminer les conséquences poétiques du « modèle Netflix » sur l'économie narrative de la série (se démarque-t-elle radicalement des séries diffusées suivant un rythme hebdomadaire ?), aussi bien que ses conséquences symboliques, en s'attardant sur les discours tenus par les différentes instances du pôle de la production afin de justifier la pertinence d'un tel mode de diffusion (revendication d'un héritage romanesque, légitimation d'une pratique de réception jusqu'alors dévalorisée : le « binge-watching»).

This article aims to analyze the segmentation into chapters in a Netflix original series, House of Cards (2013-2018). It questions how the use of the notion of chapter is linked to the broadcasting context of the series, via a streaming platform releasing all episodes at once, and thus abandoning the weekly hiatus: the audiences are then free to choose their own pace of viewing, as they can choose their pace of reading with a novel. We will seek to determine the poetic consequences of the "Netflix model" on the narrative economy of the series (does it radically differ from weekly broadcast series?), as well as its symbolic consequences, by focusing on the rhetoric issuing from various production members in order to justify the relevance of such a delivery mode (by claiming a novel's inheritance, or by legitimizing a reception practice that has been depreciated until then: binge-watching).

\section{INDEX}

Mots-clés : narratologie, chapitre, série télévisée, Netflix, vidéo à la demande, streaming, bingewatching, feuilleton

Keywords : narratology, chapter, television series, Netflix, Video on Demand, streaming, bingewatching, serial

\section{AUTEUR}

\section{ANAÏS GOUDMAND}

Université de Lausanne 